\title{
RANDOM MICROSCOPIC MODEL OF QUATERNARY ALLOYS*
}

\author{
J. KonIOR, J. LAŻEWSKI ${ }^{\dagger}$ AND A. KISIEL
}

Institute of Physics, Jagiellonian University, Reymonta 4, 30-059 Kraków, Poland

A random model of microscopic structure of zinc-blende type $\mathrm{A}_{1-x} \mathrm{~B}_{x} \mathrm{C}_{1-y} \mathrm{D}_{y}$ quaternary alloys, based entirely on the elastic bond-strètching and bond-bending forces of two-element components $\mathrm{AC}, \mathrm{AD}, \mathrm{BC}$, and $\mathrm{BD}$, was constructed. The model was applied to $\mathrm{Cd}_{1-x} \mathrm{Mn}_{x} \mathrm{Te}_{1-y} \mathrm{Se}_{y}$ quaternary alloys and the calculated nearest-neighbor distances were compared with the experimental data. Additionally, a possibility of particle exchange (within each sublattice) was included into the model and a possibility of studying the preferential coordination was discussed.

PACS numbers: $61.66 . \mathrm{Dk}, 61.72 . \mathrm{Vv}$

We consider a zinc-blende quaternary alloy $\mathrm{A}_{1-x} \mathrm{~B}_{x} \mathrm{C}_{1-y} \mathrm{D}_{y}$, in which ions of type A and B occupy one FCC sublattice and ions of type C and D occupy the second FCC sublattice. We have generalized the model of Czyżyk et al. [1] to the case of such quaternary alloys. The basic assumption of this model is that the local structure of an alloy is determined by elastic bond-stretching and bond-bending forces.

According to the Keating formula [2], the elastic energy Hamiltonian may be written as

$$
H_{\text {elastic }}=\sum_{\langle i, j\rangle} \alpha_{i j}\left(\boldsymbol{X}_{i j} \cdot \boldsymbol{X}_{i j}-x_{i j}^{2}\right)^{2}+\sum_{\{i, j, k\rangle} \beta_{i j k}\left(\boldsymbol{X}_{i j} \cdot \boldsymbol{X}_{j k}+\frac{1}{3} x_{i j} x_{j k}\right)^{2},
$$

where $\boldsymbol{X}_{i j}$ is a vector between nearest-neighbor (NN) ions $i$ and $j, \alpha_{i j}$ and $\beta_{i j k}$ are the corresponding bond-stretching and bond-bending force constants, respectively. The summations denoted with $\langle i, j\rangle$ and $\langle i, j, k\rangle$ run over all NN pairs and triplets of ions, respectively, with $\langle i, j\rangle$ and $\langle j, k\rangle$ being the NN and $i \neq k$. Furthermore, $x_{i j}$ is the equilibrium NN distance for two component systems. Finally, the bond-bending force constants $\left(\beta_{i j k}\right)$ for quaternaries were constructed from the corresponding two-component $\beta_{i j}$ with the use of the pseudocrystal approximation, i.e., $\beta_{i j k}=\sqrt{\beta_{i j} \beta_{j k}}$.

We minimized the elastic energy Hamiltonian (1) by a standard Monte Carlo procedure for a cube of $6 \times 6 \times 6=216$ cubic elementary cells, i.e., for a system of

*Supported by the Committee for Scientific Research grant no. PB-1147/P3/94/06.

$\dagger$ Also at the Institute of Nuclear Physics, Radzikowskiego 152, 31-342 Kraków, Poland. 
$N=864$ primitive elementary cells with periodic boundary conditions. Since each primitive elementary cell is occupied by one particle of the first sublattice ( $A$ or $B$ type) and by one particle of the second sublattice ( $C$ or D type), we have

$$
N_{\mathrm{A}}+N_{\mathrm{B}}=N_{\mathrm{C}}+N_{\mathrm{D}}=N \text {, }
$$

and varying the numbers $N_{\mathrm{A}}$ and $N_{\mathrm{C}}$ (and, accordingly, $N_{\mathrm{B}}$ and $N_{\mathrm{D}}$ ), we were able to study quaternary alloys with different compositions, i.e., $\mathrm{A}_{1-x} \mathrm{~B}_{x} \mathrm{C}_{1-y} \mathrm{D}_{y}$, where $x=N_{\mathrm{B}} / N$ and $y=N_{\mathrm{D}} / N$.

The simulation starts by randomly placing the particles into the positions of the corresponding sublattices. This means that $N_{\mathrm{A}}$ particles are placed at $N_{\mathrm{A}}$ randomly chosen positions of the first sublattice, while $N_{\mathrm{B}}$ particles are put at the remaining $N_{\mathrm{B}}=N-N_{\mathrm{A}}$ sites of the same sublattice. The same applies to $N_{\mathrm{C}}$ particles of the C-type and $N_{\mathrm{D}}$ particles of the D-type, but placed in the second sublattice. Having the particles placed, the elastic energy $H_{\text {elastic }}$ [Eq. (1)] is calculated for this starting configuration.

The simulation proceeds as follows. A particle is randomly chosen from the first sublattice. Then, three random numbers, all from the $[0,1]$ interval, are generated and, then, a vector is generated out of these numbers by multiplying them by $d_{\max }-$ a maximum allowed shift along one axis. With this vector, the chosen particle is moved to a new position. With a moved particle, the value of the elastic energy is calculated, yielding $H_{\text {elastic }}^{\prime}$. Then, if $H_{\text {elastic }}^{\prime}<H_{\text {elastic }}$, the new position is accepted and we go to a next step. In this case also, the substitution $H_{\text {elastic }}=H_{\text {elastic }}^{\prime}$ is made. If there is no elastic energy decrease, the particle is returned to its original position, and we go to a next step. The next step consists of randomly choosing a particle from the second sublattice, moving it, calculating a new value of the elastic energy, and accepting or rejecting the move. One iteration consists of $N$ such two-particle moves, in each one particle from the first and one particle from the second sublattice are moved. After tens of thousands of iterations, we notice that the elastic energy is not changing within the assumed accuracy. This means that the "equilibrium" was reached. The iterations which lead from the starting zinc-blende configuration to the "equilibrium" are rejected in the sense that during these iterations the structural properties of the system are not calculated. It is only after the "equilibrium" was reached, the structural properties are evaluated. To do so, we make next several tens thousands of "valid" iterations, during which the radial distribution functions are calculated. From them the NN distances are extracted.

This model was applied to $\mathrm{Cd}_{1-x} \mathrm{Mn}_{x} \mathrm{Te}_{1-y} \mathrm{Se}_{y}$ crystals. Lattice and force constants of binary compounds crystallizing in zinc-blende structure were taken from Refs. [3] and [4], respectively. Force constants of CdSe were approximated on the basis of the scheme given by Martin [5] with the use of elastic constants for zinc-blende CdSe [6]. Table contains the comparison of calculated NN distances for chosen compositions of dopants with experimental results (values in brackets) [7]. From the presented results, one can conclude that there is a good overall agreement between the calculated and measured NN distances.

The obtained results lead to a conclusion that random model presented in this paper can describe NN distances very well. It means that distances are rather 
TABLE

Calculated and experimental (in brackets) NN distances as a function of composition $x$ and $y$.

\begin{tabular}{c|c|c|c|c}
\hline \hline \multicolumn{2}{l|}{ Composition } & \multicolumn{3}{|c}{ NN distances $[\AA]$} \\
\hline$x$ & $y$ & $\mathrm{Cd}-\mathrm{Se}$ & $\mathrm{Mn}-\mathrm{Se}$ & $\mathrm{Mn}-\mathrm{Te}$ \\
\hline 0.05 & 0.10 & $2.645(2.66 \pm 0.02)$ & $2.570(2.58 \pm 0.03)$ & $2.743(2.80 \pm 0.03)$ \\
0.10 & 0.10 & $2.644(2.65 \pm 0.02)$ & $2.566(2.58 \pm 0.04)$ & $2.742(2.79 \pm 0.02)$ \\
0.15 & 0.10 & $2.642(2.65 \pm 0.02)$ & $2.564(2.58 \pm 0.03)$ & $2.740(2.78 \pm 0.02)$ \\
0.10 & 0.20 & $2.642(2.64 \pm 0.01)$ & $2.561(2.56 \pm 0.03)$ & $2.739(2.80 \pm 0.02)$
\end{tabular}

independent of small deviations from randomness observed in coordination numbers in these compounds [8]. The same conclusion can also be drawn from experimental data for InGaSbAs [9] and CdMnTeSe [8] where, for preferential coordinations, the linear dependence of distances versus dopant concentration was observed. Unfortunately, for the reasons we discuss below, we cannot study these preferences in coordination, which are detected in experiment.

Even more likely than in three component alloys $A_{1-x} B_{x}[10]$, in four-component systems the possibility of phase separation should be considered. In the simplest case of considering only the NN pairs, it would mean that out of four possible sets, $\mathrm{AC}, \mathrm{AD}, \mathrm{BC}$, and $\mathrm{BD}$, some are preferred in a sense that their presence is detected more often than it follows from pure random considerations. These random arguments yield the following values: $(1-x)(1-y)$ for $\mathrm{AC},(1-x) y$ for $\mathrm{AD}, x(1-y)$ for $\mathrm{BC}$, and $x y$ for BD pairs. If a deviation from these numbers happens, we speak about the preferences. Such preferences have been experimentally observed in InGaSbAs [8] and CdMnTeSe [9] quaternary alloys, where the $\mathrm{Ga}-\mathrm{As}$ and Mn-Se pairs are favored, respectively. We tried to study the possibility of the preferences in our model, but we have found that a simple elastic-energy model cannot account properly for the possibility of preferences. And, in our opinion, there are good reasons for that. The point is that if a possibility of exchange of two particles (of the sublattice, i.e., $A \leftrightarrow B$ and $C \leftrightarrow D$ ) arises, it immediately creates a very fast channel to separate the particles with the least preferable NN, from the elastic energy point of view. Contrary to the experimental situation, this channel is much faster than the bond-length and bond-angle relaxation, which lead to a minimum of the elastic energy. These two very different time scales are, in our opinion, responsible for the fact that a very simple picture of elastic energy minimization in the model cannot account properly for the processes which are responsible for occurrence of preferences in real systems. There is another factor related to this problem. As pointed out by some authors, the elastic energy minimization accounts rather badly for electronic states present in quaternary alloys. Actually, as it was pointed out by Islam et al. [9], the elastic energy might lead to predictions which were opposite to the experimental data.

We think that one should construct in a consistent way a model in which both the lattice relaxation (i.e., the elastic energy) and electronic states relaxation 
(by the pair approximation method, for example) are present. Such a model would be able to account for a proper description of a local structure of quaternary alloys.

\section{Acknowledgments}

One of us (J.Ł.) thanks ACC Cyfronet in Cracow for facilities CONVEX SPP1200 for doing a part of necessary calculations (grant no. KBN/IFJ/052/95).

\section{References}

[1] M.T. Czyżyk, M. Podgórny, A. Balzarotti, P. Letardi, N. Motta, A. Kisiel, M. Zimnal-Starnawska, Z. Phys. B, Condens. Matter 62, 153 (1986).

[2] P.N. Keating, Phys. Rev. 145, 637 (1966).

[3] R.W. Wyckoff, Crystal Structures, Interscience Publ., New York 1948.

[4] R.A. Mayanovic, W.-H. Pong, B.A. Bunker, Phys. Rev. B 42, 11174 (1990).

[5] R.M. Martin, Phys. Rev. B 1, 4005 (1970).

[6] Landolt-Börnstein Numerical Data and Functional Relationships in Science and Technology. New Series, Ed. K.-H. Hellwege, Group III, Vol. 11, Springer-Verlag, Berlin 1979, p. 26.

[7] A. Kisiel, J. Konior, J. Łażewski, M. Zimnal-Starnawska, E. Burattini, A. Mycielski, in preparation.

[8] A. Kisiel, J. Łażewski, M. Zimnal-Starnawska, E. Burattini, A. Mycielski, Acta Phys. Pol. A 90, 1032 (1996).

[9] S.M. Islam, B.A. Bunker, Phys. Lett. A 156, 247 (1991).

[10] P. Boguslawski, A. Baldareschi, Solid State Commun. 66, 679 (1988). 